\title{
A CONCENTRAÇÃO DO CONTROLE ACIONÁRIO E A POLÍTICA DE DIVIDENDOS DAS EMPRESAS LISTADAS NA BOVESPA: UMA ABORDAGEM EXPLORATÓRIA À LUZ DA TEORIA DE AGÊNCIA
}

\section{THE SHAREHOLDING CONCENTRATION AND THE DIVIDEND POLICY OF COMPANIES LISTED ON BOVESPA: AN EXPLORATORY APPROACH IN THE LIGHT OF AGENCY THEORY}

\author{
FLÁVIA ZÓBOLI DALMÁCIO \\ Doutoranda em Controladoria e Contabilidade pela \\ Universidade de São Paulo \\ Pesquisadora e Professora da Fundação Instituto Capixaba \\ de Pesquisas em Contabilidade, Economia e Finanças \\ E-mail: flavia@fucape.br
}

\author{
LUIZ JOÃO CORRAR \\ Professor Doutor da Faculdade de Economia, \\ Administração e Contabilidade da \\ Universidade de São Paulo \\ E-mail: ljcorrar@usp.br
}

\section{Resumo}

Este artigo busca, sob a perspectiva da Teoria de Agência e a partir de revisão bibliográfica, pesquisa empírica exploratória e investigações analíticas, verificar se a composição acionária das empresas brasileiras com ações negociadas na Bovespa, tem relação com a política de dividendos por elas adotada. Os resultados obtidos, por meio de testes estatísticos, sugerem a não rejeição da hipótese $\left(\mathrm{H}_{0}\right)$ elaborada neste trabalho. Verificou-se que um aumento na concentração acionária provoca um aumento no valor dos dividendos pagos por ação (ON), entretanto, há uma expectativa de que aquele aumento provoque uma diminuição significativa no percentual do payout. Portanto, constatam-se indícios de que a composição acionária das empresas brasileiras possa explicar, em partes, a política de dividendos por elas adotada. Vale ressaltar que outros fatores podem influenciar os resultados empíricos como, por exemplo, a regulamentação brasileira sobre a política de dividendos e juros sobre o capital próprio, que não foram consideradas como variáveis neste trabalho.

Palavras-chave: controle acionário; política de dividendos; Bovespa; mercado brasileiro; Teoria de Agência.

\begin{abstract}
From the perspective of Agency Theory, this paper seeks to verify if the shareholder composition of the Brazilian companies which have shares negotiated on Bovespa, are related to the dividend policy adopted by these companies. This work was developed from a bibliographic review and, based on an exploratory empirical research, there had been made analytical investigations. The results, obtained by statistic tests, suggest the non rejection of the hypothesis $(\mathrm{HO})$ developed in this work. It was verified that growth of shareholding concentration causes an increase on the dividends paid per share (ON). However, there is an expectation that an increase on shareholding concentration provokes a significant reduction on payout percentage. Hence, it follows that there is evidence that Brazilian companies' shareholding composition can, in part, explain the dividend policy adopted by the studied companies. It is important to emphasize that other factors may influence the empirical results as, for instance, Brazilian regulation about dividends and interest policy about own capital, which had not been considered as variables in this study.
\end{abstract}

Key words: shareholding, dividend policy, Bovespa, Brazilian market, Agency Theory. 


\section{INTRODUÇÃO}

No Brasil, existe uma alta concentração de capital votante em poder de um número reduzido de acionistas. Diante desse contexto, como a decisão sobre a distribuição de dividendos é tomada pela administração, o pagamento deles acontece apenas para atender à legislação societária, gerando a expectativa de que a parte não distribuída é utilizada no financiamento de novos investimentos (reinvestimentos); o que aumenta o valor da empresa e, consequentemente, o das ações. Há portanto, algum relacionamento entre dividendos e concentração acionária. De acordo com Lopes (2002, p. 77-78):

A concentração do controle acionário no Brasil é bastante grande. Pesquisa realizada pela Empresa Economática e publicada no jornal Gazeta Mercantil (22 dez. 2000, p. C-1) mostra que "... na maior parte das companhias que têm ações ordinárias, mais de $70 \%$ do capital está em poder de um único acionista”.

[...] Em empresas com poucos acionistas (no caso brasileiro, em muitas empresas o controle acionário está nas mãos de um único acionista), a contabilidade perde o seu papel de redutora de assimetria de informação entre acionistas e gestores. Nessas empresas, o acionista majoritário possui acesso privilegiado às informações gerenciais da empresa, [...].

[...], percebe-se que os acionistas majoritários têm praticado 'abusos' em relação aos minoritários.

Diante do exposto, percebe-se que empresas com poucos acionistas, normalmente, têm grande influência na administração, devido à grande concentração do capital votante. E dessa influência, podem surgir relações de agência. Segundo Brigham, Gapenski e Ehrhardt (2001, p. 39):

Uma relação de agência surge quando um ou mais indivíduos, chamados principais, (1) contratam outro indivíduo ou organização, chamado agente, para realizar algum tipo de serviço e (2) estes então delegam autoridade de tomada de decisões para aquele agente.

$\mathrm{Na}$ relação de agência, o principal delega poderes para que o agente gerencie seus recursos. Nessa relação, espera-se que o agente tome decisões que visem ao atendimento dos interesses do principal. No entanto, se houver concentração do controle acionário em uma empresa, os administradores/ gestores, provavelmente, serão acionistas ordinários e, presumivelmente, operarão a fim de maximizar seu próprio bem estar, medido pelo aumento de sua riqueza pessoal (aumento do patrimônio da empresa e conseqüente valorização de suas ações).

Diante desse argumento, surge a seguinte questão de pesquisa: Qual é a relação da política de dividendos adotada pelas empresas brasileiras com as ações negociadas na Bovespa e a forma de composição acionária (concentração do capital votante) dessas empresas?

Uma suposta resposta ao problema a ser investigado, dadas as premissas da Teoria de Agência, é que nas empresas cujo controle acionário (poder de decisão) é privilégio de poucos e onde não há uma separação entre a propriedade e a administração, os administradores/ acionistas ordinários tendem a reter lucros e não distribuir dividendos, para maximizar sua riqueza pessoal. Para tanto, elaboram-se as seguintes hipóteses: 
- $\mathrm{H}_{0}$ : No Brasil, a concentração do controle acionário das empresas influencia a distribuição de dividendos; e

- $\mathrm{H}_{1}$ : No Brasil, a concentração do controle acionário das empresas não influencia a distribuição de dividendos.

Portanto, diante dessas hipóteses que serão testadas e analisadas, o objetivo principal deste trabalho é identificar se a composição acionária das empresas brasileiras, com ações negociadas na Bovespa, tem relação com a política de dividendos por elas adotada.

Num primeiro momento, realiza-se uma pesquisa bibliográfica, para entender os fundamentos da Teoria de Agência e enfatizar os principais conflitos decorrentes das suas relações; depois, concretizam-se uma pesquisa empírica exploratória e investigações analíticas, para relacionar aquela teoria à política de dividendos.

De maneira específica, estimaram-se regressões no período de 1998 a 2005, e utilizou-se o $\mathrm{R}^{2}$ como métrica para avaliar o poder explanatório das variáveis: dividendos pagos por ação e payout ${ }^{1}$, considerando as empresas com ações ordinárias com direito a voto.

\section{POLÍTICA DE DIVIDENDOS}

De acordo com Loss e Sarlo Neto (2003, p. 40), a política de dividendos implica na decisão de quanto deverá ser distribuído ao acionista e, conseqüentemente, quanto ficará retido.

Entre as teorias desenvolvidas sobre a política de dividendos, destacam-se: a Teoria da Irrelevância dos Dividendos (MODIGLIANI e MILLER, 1961); a Teoria do Pássaro na Mão (LINTNER, 1962 e GORDON, 1963); e a Teoria da Preferência Tributária.

Modigliani e Miller (1961), de acordo com uma série de premissas, afirmam que o valor da empresa não é afetado pela distribuição de dividendos, mas, pela capacidade de geração de valor e risco de seus ativos. Para esses autores, inexiste uma estrutura financeira ótima entre capital próprio e de terceiros que vá impactar, positivamente, o valor das ações.

Para Lintner (1962) e Gordon (1963), num mundo de incertezas, os investidores preferirão receber seus dividendos, o mais breve possível. Para esses autores, existe uma relação direta entre a política de distribuição de dividendos da empresa e o seu valor de mercado. De acordo com a Teoria do Pássaro na Mão ou a Teoria da Relevância dos Dividendos, o pagamento de dividendos correntes reduz a incerteza dos investidores, elevando o preço das ações da empresa.

Segundo a Teoria da Preferência Tributária, os investidores preferem que as empresas retenham lucros do que distribuam dividendos, pois os ganhos de capital de longo prazo estão sujeitos a impostos menos onerosos.

No Brasil, muitos estudos foram realizados sobre a política de dividendos adotada e, conseqüentemente, sobre qual teoria é aplicada nas empresas brasileiras. No entanto, o

\footnotetext{
${ }^{1}$ Dividendos propostos na DOAR $\div$ Lucro Líquido do Exercício.
} 
foco deste trabalho é estudar o conflito de interesses que pode existir no caso de concentração acionária.

"Como a política de dividendos é estabelecida pela administração das entidades e, por seu turno, os lucros retidos estão sob o seu controle" (LOSS e SARLO NETO, 2003, p. 45), busca-se verificar a política de pagamento de dividendos, na existência de concentração de capital votante (concentração acionária).

\section{TEORIA DE AGÊNCIA E POLÍTICA DE DIVIDENDOS}

Os conflitos de interesse entre administradores e acionistas, inicialmente, foram tratados por Smith (1776 apud JENSEN e MECKLING, 1976), na obra “A riqueza das nações". Berle e Means (1932) apresentam evidências empíricas sobre a concentração de propriedade e abordam questões a respeito da separação do direito de propriedade e controle do capital.

Ross (1973) destacou que a relação de agência é uma das mais antigas e um dos hábitos mais comuns da interação social, além de caracterizar vários modelos de relação entre principal e agente. Jensen e Meckling (1976) procuram dar um novo enfoque aos estudos existentes até então sobre relacionamentos de agência, aprofundando-se nos conflitos das relações contratuais e acrescentando, prioritariamente, alguns aspectos comportamentais relacionados à administração. Além disso, definem o conceito de custos de agência, investigam a natureza desses custos e mostram a relação deles com a "separação do direito de propriedade e controle do capital".

Jensen e Meckling (1976) formalizam econômica e matematicamente a Teoria de Agência, utilizando o conceito da Curva de Utilidade. Eisenhardt (1989) a tratou como uma importante, mas controversa teoria e expôs que, em geral, ela é a relação que espelha a estrutura básica de agency de um principal e um agente. A intenção do trabalho de Eisenhardt é clarificar algumas confusões a respeito da Teoria de Agência e incentivar estudos organizacionais com aplicações mais amplas a seu respeito.

A Teoria de Agência busca explicar a relação entre dois ou mais indivíduos. Segundo Hendriksen e van Breda (1999, p. 139), "um desses dois indivíduos é um agente do outro, chamado de principal - daí o nome de teoria de agency. 0 agente compromete-se a realizar certas tarefas para o principal; o principal compromete-se a remunerar o agente".

Nessa relação, espera-se que o agente tome decisões que visem aos interesses do principal, entretanto, de acordo com Eisenhardt (1989, p. 59), principal e agente estão engajados em um comportamento coorporativo, mas possuem diferentes metas e diferentes atitudes com relação ao risco.

Jensen e Meckling (1976, p. 5) definem uma relação de agência como "um contrato pelo qual uma ou mais pessoas, em que o (s) principal(is) contrata $(\mathrm{m})$ outra pessoa (o agente) para executar algum serviço em favor deles e que envolva delegar, ao agente, alguma autoridade de tomada de decisão" (tradução nossa).

A Teoria de Agência, apresentada por Jensen e Meckling (1976), defende que é quase impossível assegurar que o agente tome a melhor decisão do ponto de vista do principal. Assim, ao principal destina-se o maior lucro possível e não mais o lucro máximo. Vale ressaltar que "o administrador-proprietário de uma empresa sempre procura maximizar sua utilidade. Sendo assim, se ele for o único proprietário, supõe-se que ele procure maximizar a riqueza da empresa (ou a sua própria)" (JENSEN e MECLING, 1976, 1011). 
A hipótese $\left(\mathrm{H}_{0}\right)$ levantada neste trabalho está sustentada, principalmente, nesse pressuposto, pois o administrador-proprietário cumpre a principal meta do administrador financeiro, ou seja, maximizar a riqueza dos acionistas (no caso, ele mesmo aumentando o patrimônio da empresa e, conseqüentemente, valorizando o valor de suas ações).

Segundo Loss (2004, p. 15):

0 mercado brasileiro possui características diferenciadas daquelas encontradas nos mercados mais desenvolvidos. No mercado brasileiro, tem havido escassez de recursos no longo prazo, as empresas são familiares, concentradas nas mãos de poucos, os custos de transação, embora tenha havido esforços no sentido de reduzi-los, são altos.

Por estes motivos, há uma expectativa de que reter lucros são decisões presentes na realidade das companhias brasileiras. Um dos motivos que podem estar conduzindo a esta retenção é a necessidade de aplicações em investimentos, dada as dificuldades em obter-se recursos de longo prazo.

Procianoy (1994, p. 8-20) verificou que, no Brasil, há uma tendência das empresas reterem a maior parcela possível dos lucros, pelo fato de haver escassez de recursos de longo prazo, ou até mesmo, ações negociadas abaixo do valor patrimonial contábil. Ou seja, verifica-se que os controladores têm um forte incentivo para reter a maior parcela possível dos lucros de suas empresas, sem distribuí-los.

O Quadro 1, apresenta uma síntese de trabalhos internacionais que tratam da aplicação da Teoria de Agência à política de dividendos.

QUADRO 1: Síntese dos trabalhos sobre a aplicação da Teoria de Agência à política de dividendos:

\begin{tabular}{|c|c|c|}
\hline $\begin{array}{c}\text { ANO DE } \\
\text { PUBLICAÇÃO }\end{array}$ & AUTOR (ES) & TÍTULO DO TRABALHO \\
\hline 1993 & Alessandro Sembenelli & $\begin{array}{c}\text { Signalling, Financial Hierarchy and Agency Theory as Explanations } \\
\text { for Dividends Behaviour: Evidence from Italian Firm Data }\end{array}$ \\
\hline 2000 & $\begin{array}{l}\text { Rafael La Porta; } \\
\text { Florêncio Lopez-De- } \\
\text { Silanes; Andrei Shleifer; } \\
\text { Robert W. Vishny }\end{array}$ & Agency Problems and Dividend Policies around the World \\
\hline 2000 & $\begin{array}{l}\text { A. Sabur Mollah; Kevin } \\
\text { Keasey; Helen Short }\end{array}$ & $\begin{array}{c}\text { The Influence of Agency Costs on Dividend Policy in an Emerging } \\
\text { Market: Evidence from the Dhaka Stock Exchange }\end{array}$ \\
\hline 2005 & $\begin{array}{l}\text { Alon Brav, John R. } \\
\text { Graham, Campbell R. } \\
\text { Harvey, Roni Michaely }\end{array}$ & Payout policy in the $21^{\text {st }}$ century \\
\hline
\end{tabular}

Fonte: Elaborado pelos autores.

Entre os trabalhos citados, destaca-se o de La Porta et. al (2000). Esses autores estudaram os problemas de agência existentes em grandes empresas de 33 países e verificaram que o sistema legal do país influencia na política de dividendos, mas que esse mecanismo não funciona bem em qualquer mercado, pois a eficácia da legislação difere entre os países. De maneira específica, eles observaram que as empresas que pagam mais dividendos são aquelas que funcionam em países onde existe maior proteção legal aos acionistas minoritários. No entanto, essas empresas, quando em crescimento, diminuem os dividendos, porque os acionistas (protegidos por lei) estão dispostos a esperar o retorno dos investimentos futuros. Por outro lado, investidores sem proteção legal preferem receber dividendos, mesmo existindo boas perspectivas para o futuro. 


\section{METODOLOGIA}

Com o objetivo de analisar o poder explicativo da variável concentração acionária e testar a validade das hipóteses levantadas, o estudo fundamenta-se na Teoria de Agência. $\mathrm{E}$, por meio de uma pesquisa exploratória, empírico-analítica, pretende relacionar essa teoria à política de distribuição de dividendos adotada pelas empresas brasileiras com ações ordinárias negociadas na Bovespa.

Os dados a respeito dessas empresas foram obtidos no banco de dados Economática, com abordagem aos seguintes aspectos:

- Ações ordinárias com direito a voto (ON);

- Características sobre a concentração acionária (composição acionária concentração do capital votante), ou seja, percentual das ações com direito a voto possuído pelo primeiro maior acionista; e

- Histórico da política de dividendos, ou seja, quanto cada empresa pagou de dividendos nos últimos anos (percentual dos lucros distribuídos ao longo do tempo) para ações ordinárias, ou seja, dividendo pago por ação $\$$ e payout (dividendos propostos na DOAR $\div$ lucro líquido\$ do exercício).

Foram analisadas 438 empresas, no período de 1998 a 2005. Aquelas que não possuíam informações sobre a concentração acionária, dividendos pagos por ação ou payout, foram eliminadas da amostra, bem como os outliers.

Como a amostra, a princípio, não demonstrou características de normalidade, utilizou-se o teste de Jarque-Bera, para adequação e atendimento dos pressupostos estatísticos. As variáveis foram transformadas (Log).

Portanto, por meio de análise de regressão linear, buscou-se a relação entre os dividendos (pagos por ação e payout) e a composição acionária, ou seja, foi utilizada uma análise de regressão linear simples, para verificar se a composição acionária (variável independente) influencia o pagamento de dividendos (variável dependente). Os dividendos foram analisados por meio dos dois modelos descritos a seguir:

- Dividendos pagos por ação da empresa; e

+ Payout.

$$
\text { Modelo (1): } \log \left(D A_{i j}\right)=B_{0}+B_{1} \log \left(C A_{i j}\right)+\varepsilon_{i j}
$$

Em que:

$\mathrm{DA}_{\mathrm{ij}}=$ dividendos pagos por ação da empresa i no ano j (ajustados pela inflação do período)

$B_{0}=$ coeficiente de interseção

$B_{1}=$ coeficiente de variação e/ou inclinação

$\mathrm{CA}_{\mathrm{ij}}=$ composição acionária da empresa $\mathrm{i}$ no ano $\mathrm{j}$

$\varepsilon_{\mathrm{ij}}=$ termo de erro da regressão

Modelo (2): $\log \left(P O_{i j}\right)=B_{0}+B_{1} \log \left(C A_{i j}\right)+\varepsilon_{i j}$ 
Em que:

$\mathrm{PO}_{\mathrm{ij}}=$ payout da empresa $\mathrm{i}$ no ano $\mathrm{j}$

$B_{0}=$ coeficiente de interseção

$B_{1}=$ coeficiente de variação e/ou inclinação

$\mathrm{CA}_{\mathrm{ij}}=$ composição acionária da empresa $\mathrm{i}$ no ano $\mathrm{j}$

$\varepsilon i j$ = termo de erro da regressão

Verificou-se a correlação entre as variáveis, e uma vez observada a correlação linear significativa, realizou-se a análise de regressão. A partir dela, foram analisados os $R^{2}$ (poder explicativo da variável composição acionária) e os interceptos.

No entanto, como alguns resultados, obtidos por meio da regressão linear simples, não se demonstraram consistentes e, estatisticamente, significantes no que se refere à relação entre a política de dividendos adotada pelas empresas e a forma de composição acionária (concentração do capital votante), buscou-se investigar os dados em modelos de regressão, em painel, a fim de acompanhar as empresas ao longo do tempo.

Ressalta-se, de acordo com Gujarati (2006, p. 514), que os dados em painel, em relação aos dados em corte transversal, "proporcionam dados mais informativos, mais variabilidade e menos colinearidade entre as variáveis, mais graus de liberdade e mais eficiência".

A utilização de dados em painel permite conjugar a diversidade de comportamentos individuais, com as dinâmicas de ajustamento, ainda que potencialmente distintas, ou seja, permite tipificar as respostas de diferentes indivíduos a determinados acontecimentos, em diferentes momentos. Além disso, a maior quantidade de informação disponível aumenta a eficiência da estimação, porque é possível identificar e medir efeitos que não serão pura e simplesmente detectáveis em estudos exclusivamente seccionais ou temporais (MARQUES, 2000).

\section{4 - ANÁLISE DOS RESULTADOS}

Foram realizadas duas regressões, a partir de dois modelos em função da concentração acionária, considerando-se: no primeiro, os dividendos por ação; e no segundo, o payout. Na construção dos modelos de regressão, foram observados os pressupostos: normalidade (Jarque-Bera), homocedasticidade (White Heteroskedasticity Test), linearidade, ausência de autocorrelação (Breusch-Godfrey Serial Correlation LM Test) e ausência de multicolinearidade.

Após a coleta dos dados, foram consideradas apenas as empresas que continham informações sobre todas as variáveis, em todos os anos analisados (1998 a 2005). Ou seja, foram incluídas, na análise, apenas as observações com dados completos. Verificou-se que, na média, $64,18 \%$ do capital dessas empresas está em poder de um único acionista, confirmando as afirmações de Lopes (2001), de que, no Brasil, a concentração acionária é grande. Na Tabela 1, apresenta-se a estatística descritiva da amostra. 
Tabela 1: Estatística descritiva da amostra

\begin{tabular}{|c|c|c|c|c|c|c|}
\hline Ano & Variáveis & $\mathbf{N}^{\circ}$ & Mínimo & Máximo & Média & Desvio Padrão \\
\hline \multirow[t]{3}{*}{1998} & Concentração Acionária (CA) & 360 & 9,62 & 100,00 & 59,64 & 25,04 \\
\hline & Dividendos Pagos por Ação (DA) & 389 & 0,00 & 24,83 & 0,43 & 2,01 \\
\hline & Payout (PO) & 316 & $-1.601,04$ & $2.349,41$ & 24,07 & 171,41 \\
\hline \multirow[t]{3}{*}{1999} & Concentração Acionária (CA) & 377 & 0,58 & 100,00 & 62,02 & 25,31 \\
\hline & Dividendos Pagos por Ação (DA) & 400 & 0,00 & $2.289,16$ & 6,16 & 114,46 \\
\hline & Payout (PO) & 335 & $-332,96$ & 815,47 & 26,23 & 73,11 \\
\hline \multirow[t]{3}{*}{2000} & Concentração Acionária (CA) & 387 & 0,58 & 100,00 & 62,82 & 25,44 \\
\hline & Dividendos Pagos por Ação (DA) & 404 & 0,00 & $2.057,72$ & 5,58 & 102,39 \\
\hline & Payout (PO) & 328 & $-471,22$ & $5.210,59$ & 42,66 & 296,57 \\
\hline \multirow[t]{3}{*}{2001} & Concentração Acionária (CA) & 393 & 9,45 & 100,00 & 62,39 & 25,30 \\
\hline & Dividendos Pagos por Ação (DA) & 412 & 0,00 & 124,76 & 1,27 & 9,05 \\
\hline & Payout (PO) & 335 & $-463,58$ & $1.994,22$ & 32,37 & 153,98 \\
\hline \multirow[t]{3}{*}{2002} & Concentração Acionária (CA) & 396 & 9,47 & 100,00 & 65,19 & 25,58 \\
\hline & Dividendos Pagos por Ação (DA) & 418 & 0,00 & $1.755,56$ & 8,59 & 110,64 \\
\hline & Payout (PO) & 336 & $-9.043,05$ & $30.053,18$ & 81,91 & $1.741,96$ \\
\hline \multirow[t]{3}{*}{2003} & Concentração Acionária (CA) & 402 & 9,47 & 100,00 & 66,06 & 25,76 \\
\hline & Dividendos Pagos por Ação (DA) & 421 & 0,00 & 82,41 & 0,58 & 4,39 \\
\hline & Payout (PO) & 340 & $-973,24$ & 870,69 & 29,81 & 93,02 \\
\hline \multirow[t]{3}{*}{2004} & Concentração Acionária (CA) & 417 & 10,10 & 100,00 & 66,32 & 25,75 \\
\hline & Dividendos Pagos por Ação (DA) & 428 & 0,00 & 581,56 & 1,92 & 28,22 \\
\hline & Payout (PO) & 358 & $-11.125,78$ & $2.007,85$ & 7,70 & 604,86 \\
\hline \multirow[t]{3}{*}{2005} & Concentração Acionária (CA) & 426 & 5,68 & 100,00 & 66,12 & 25,55 \\
\hline & Dividendos Pagos por Ação (DA) & 438 & 0,00 & 160,27 & 1,24 & 8,88 \\
\hline & Payout (PO) & 328 & $-3.635,59$ & $1.233,16$ & 35,36 & 235,44 \\
\hline
\end{tabular}

Fonte: Elaborada pelos autores.

De acordo com os dados amostrais, a respeito da concentração acionária das empresas com ações negociadas na Bovespa e os dividendos pagos por ação, a equação da reta de regressão que expressa a relação entre essas variáveis é dada pela equação demonstrada na Tabela 2 (conforme modelo 1): 
Tabela 2: Análise de regressão das variáveis Dividendo Pagos por Ação (DA) e Concentração Acionária (CA)

\begin{tabular}{|c|c|c|c|c|c|c|c|c|}
\hline \multicolumn{9}{|c|}{$\log (D A i j)=B 0+B 1 * \log (C A)+E i j$} \\
\hline \multirow{17}{*}{ 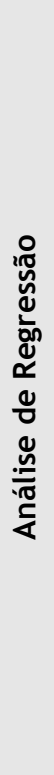 } & Ano & $\begin{array}{c}\mathrm{N}^{\circ} \mathrm{de} \\
\text { Empresas }\end{array}$ & Resultado & C & $\log (C A)$ & R2 & P-value & $\begin{array}{l}\text { Correlação de } \\
\text { Pearson }\end{array}$ \\
\hline & \multirow{2}{*}{1998} & \multirow{2}{*}{190} & Coef. & 1,382 & $-1,125$ & \multirow{2}{*}{0,0459} & \multirow{2}{*}{0,003} & \multirow{2}{*}{$-0,214$} \\
\hline & & & P-value & 0,353 & 0,003 & & & \\
\hline & \multirow{2}{*}{1999} & \multirow{2}{*}{181} & Coef. & $-0,382$ & $-0,696$ & \multirow{2}{*}{0,0196} & \multirow{2}{*}{0,060} & \multirow{2}{*}{$-0,140$} \\
\hline & & & P-value & 0,794 & 0,060 & & & \\
\hline & \multirow{2}{*}{2000} & \multirow{2}{*}{188} & Coef. & $-2,109$ & $-0,266$ & \multirow{2}{*}{0,0025} & \multirow{2}{*}{0,493} & \multirow{2}{*}{$-0,050$} \\
\hline & & & P-value & 0,172 & 0,493 & & & \\
\hline & \multirow{2}{*}{2001} & \multirow{2}{*}{194} & Coef. & 1,131 & $-0,973$ & \multirow{2}{*}{0,0220} & \multirow{2}{*}{0,039} & \multirow{2}{*}{$-0,149$} \\
\hline & & & P-value & 0,551 & 0,039 & & & \\
\hline & \multirow{2}{*}{2002} & \multirow{2}{*}{183} & Coef. & $-0,380$ & $-0,592$ & \multirow{2}{*}{0,0080} & \multirow{2}{*}{0,230} & \multirow{2}{*}{$-0,089$} \\
\hline & & & $P$-value & 0,849 & 0,230 & & & \\
\hline & \multirow{2}{*}{2003} & \multirow{2}{*}{176} & Coef. & 0,173 & $-0,756$ & \multirow{2}{*}{0,0165} & \multirow{2}{*}{0,090} & \multirow{2}{*}{$-0,128$} \\
\hline & & & $P$-value & 0,924 & 0,090 & & & \\
\hline & \multirow{2}{*}{2004} & \multirow{2}{*}{197} & Coef. & $-0,693$ & $-0,474$ & \multirow{2}{*}{0,0062} & \multirow{2}{*}{0,268} & -0079 \\
\hline & & & $P$-value & 0,690 & 0,268 & & & $-0,0 / Y$ \\
\hline & 2005 & 212 & Coef. & 0,061 & $-0,615$ & 0119 & 0113 & -0109 \\
\hline & & & $P$-value & 0,969 & 0,113 & 0,0117 & 0,110 & $-0,107$ \\
\hline
\end{tabular}

Fonte: Elaborada pelos autores.

Na Tabela 2, apresentam-se: os coeficientes $\beta_{0}(C)$ e $\beta_{1}$ (LogCA) e os seus respectivos $p$-values; o coeficiente de determinação $\left(R^{2}\right)$ e seu $p$-value; e o coeficiente de correlação de Pearson, obtidos em cada um dos anos analisados, por meio da análise de regressão linear simples. Vale ressaltar que o número de empresas variou, em cada ano, devido à disponibilidade completa dos dados utilizados na análise.

Analisando o coeficiente $\operatorname{LogCA}$, verifica-se que se a concentração acionária varia em uma unidade, os dividendos pagos por ação variam de forma inversa, ou seja, um aumento daquela provoca uma diminuição desses, no entanto, os coeficientes nos anos de 2000, 2002, 2004 e 2005 não são, estatisticamente, significativos (considerando-se a igual a $10 \%)$. Acrescenta-se que, em todos os anos, existe uma fraca correlação negativa entre a variável Concentração Acionária (LogCA) e a variável Dividendo pago por Ação (LogDA).

O valor de $R^{2}$, em todos os anos, é baixo, significando que a variável explicativa Concentração Acionária (LogCA) responde por uma pequena variação do Dividendo pago por ação (LogDA); o que indica que o modelo não está ajustado aos dados. Além disso, verifica-se que o modelo não é, estatisticamente, significativo (em nível de 10\%), para os anos de 2000, 2002, 2004 e 2005.

De acordo com os dados amostrais, a respeito da concentração acionária das empresas com ações negociadas na Bovespa e o payout dos dividendos, a equação da reta de regressão que expressa a relação entre essas variáveis é dada pela equação demonstrada na Tabela 3 (conforme modelo 2). 
Tabela 3: Análise de regressão das variáveis Payout (PO) e Concentração Acionária (CA)

\begin{tabular}{|c|c|c|c|c|c|c|c|c|}
\hline \multicolumn{9}{|c|}{$\log (P o i j)=B 0+B 1 * \log (C A)+E i j$} \\
\hline \multirow{17}{*}{ 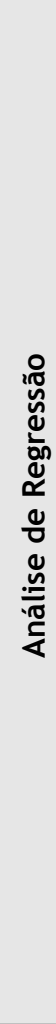 } & Ano & $\begin{array}{c}\text { N. de } \\
\text { Empresas }\end{array}$ & Resultado & C & $\log (C A)$ & R2 & $P$-value & $\begin{array}{c}\text { Correlação de } \\
\text { Pearson }\end{array}$ \\
\hline & \multirow{2}{*}{1998} & \multirow{2}{*}{164} & Coef. & 4,075 & $-0,069$ & \multirow{2}{*}{0,0035} & \multirow{2}{*}{0,449} & \multirow{2}{*}{$-0,060$} \\
\hline & & & $P$-value & 0,000 & 0,449 & & & \\
\hline & \multirow{2}{*}{1999} & \multirow{2}{*}{163} & Coef. & 4,469 & $-0,181$ & \multirow{2}{*}{0,0212} & \multirow{2}{*}{0,064} & \multirow{2}{*}{$-0,146$} \\
\hline & & & $P$-value & 0,000 & 0,064 & & & \\
\hline & \multirow{2}{*}{2000} & \multirow{2}{*}{182} & Coef. & 3,612 & 0,038 & \multirow{2}{*}{0,0007} & \multirow{2}{*}{0,714} & \multirow{2}{*}{0,027} \\
\hline & & & $P$-value & 0,000 & 0,714 & & & \\
\hline & \multirow{2}{*}{2001} & \multirow{2}{*}{176} & Coef. & 4,303 & $-0,135$ & \multirow{2}{*}{0,0054} & \multirow{2}{*}{0,332} & \multirow{2}{*}{$-0,074$} \\
\hline & & & $P$-value & 0,000 & 0,332 & & & \\
\hline & \multirow{2}{*}{2002} & \multirow{2}{*}{144} & Coef. & 5,386 & $-0,405$ & \multirow{2}{*}{0,0436} & \multirow{2}{*}{0,012} & \multirow{2}{*}{$-0,209$} \\
\hline & & & $P$-value & 0,000 & 0,012 & & & \\
\hline & \multirow{2}{*}{2003} & \multirow{2}{*}{184} & Coef. & 3,768 & $-0,001$ & \multirow{2}{*}{0,0000} & \multirow{2}{*}{0,993} & \multirow{2}{*}{$-0,001$} \\
\hline & & & $P$-value & 0,000 & 0,993 & & & \\
\hline & \multirow{2}{*}{2004} & \multirow{2}{*}{207} & Coef. & 3,548 & 0,054 & \multirow{2}{*}{0,0012} & \multirow{2}{*}{0,627} & דר? \\
\hline & & & $P$-value & 0,000 & 0,627 & & & 0,034 \\
\hline & & & Coef. & 3,568 & 0,083 & & & \\
\hline & 2005 & 200 & $P$-value & 0,000 & 0,416 & 0,0033 & 0,416 & 0,058 \\
\hline
\end{tabular}

Fonte: Elaborada pelos autores.

Na Tabela 3, apresentam-se: os coeficientes $\beta_{0}(C), \beta_{1}$ (LogPO) e os seus respectivos $p$-values; o coeficiente de determinação $R^{2}$ e seu $p$-value; e o coeficiente de correlação de Pearson, obtidos em cada um dos anos analisados, por meio da análise de regressão linear simples.

Em análise do coeficiente LogCA, verifica-se que se a concentração acionária, nos anos de 1998, 1999, 2001, 2002 e 2003, varia em uma unidade, o payout varia de forma inversa, ou seja, um aumento daquela provoca uma diminuição desse, no entanto, os coeficientes nos anos de 1998, 2001 e 2003 não são, estatisticamente, significativos (considerando-se a igual a 10\%). Acrescenta-se que, em todos os anos, existe uma fraca correlação negativa entre a variável Concentração Acionária (LogCA) e a variável Payout (LogPO).

O valor de $R^{2}$, em todos os anos, é baixo, significando que a variável explicativa Concentração Acionária (LogCA) responde por uma pequena variação do Payout (LogPO); o que indica que o modelo não está ajustado aos dados. Além disso, verifica-se que o modelo só é, estatisticamente, significativo (em nível de 10\%), no ano de 1999.

A fim de enriquecer a análise empírica, conforme motivos comentados anteriormente (dados mais informativos, mais variabilidade e menos colinearidade entre as variáveis etc), as variáveis CA, DA e PO foram analisadas por meio de regressão em painel (efeito combinado pelo Método GLS). Na análise, foram utilizadas as mesmas 438 empresas 
brasileiras $^{2}$ com ações ordinárias negociadas na Bovespa, durante o período de 1998 a 2005.

De acordo com os dados da Tabela 4, verifica-se que foi feita a estimação de regressão por pesos nas cross-sections, pelo método dos Mínimos Quadrados Generalizados. Quando considerada a equação $\mathbf{D A}=\boldsymbol{\beta}_{0}+\boldsymbol{\beta}_{1} \mathbf{C A}+\boldsymbol{\varepsilon}$, ou seja, CA e DA como variáveis dependentes, observa-se que o poder explicativo da regressão $R^{2}$ é de 21 , $59 \%$, considerando-se um nível de significância de $5 \%$, ou seja, a concentração acionária explica $21,59 \%$ do comportamento dos dividendos pagos por ação. Além disso, verifica-se que a regressão e os seus coeficientes são, estatisticamente, significantes ( $\alpha$ igual a 5\%).

Tabela 4: Output para regressão com efeito combinado pelo método GLS, considerando Dividendo por Ação (DA) como a variável dependente

\begin{tabular}{|c|c|c|c|c|}
\hline \multicolumn{4}{|c|}{ Dependent Variable: DA? } & \\
\hline \multicolumn{4}{|c|}{ Method: Pooled EGLS (Cross-section weights) } & \\
\hline \multicolumn{3}{|l|}{ Sample: 19982005} & & \\
\hline \multicolumn{3}{|c|}{ Included observations: 8} & & \\
\hline \multicolumn{3}{|c|}{ Cross-sections included: 426} & & \\
\hline \multicolumn{4}{|c|}{ Total pool (unbalanced) observations: 3143} & \\
\hline \multicolumn{5}{|c|}{ Linear estimation after one-step weighting matrix } \\
\hline \multicolumn{5}{|c|}{ White diagonal standard errors \& covariance (d.f. corrected) } \\
\hline \multicolumn{5}{|c|}{ Cross sections without valid observations dropped } \\
\hline Variable & Coefficient & Std. Error & t-Statistic & Prob. \\
\hline C & -0.604396 & 0.232984 & -2.594157 & 0.0095 \\
\hline \multirow[t]{2}{*}{ CA? } & 0.021734 & 0.007424 & 2.927447 & 0.0034 \\
\hline & \multicolumn{2}{|c|}{ Weighted Statistics } & & \\
\hline R-squared & 0.216115 & \multicolumn{2}{|c|}{ Mean dependent var } & 10.39634 \\
\hline Adjusted R-squared & 0.215865 & \multicolumn{2}{|c|}{ S.D. dependent var } & 40.76843 \\
\hline S.E. of regression & 36.10102 & \multicolumn{2}{|c|}{ Sum squared resid } & 4093613. \\
\hline F-statistic & 865.9631 & \multicolumn{2}{|c|}{ Durbin-Watson stat } & 0.661529 \\
\hline Prob(F-statistic) & 0.000000 & & & \\
\hline
\end{tabular}

Considerando os dados da Tabela 4, observa-se que se a concentração acionária varia em uma unidade, os dividendos pagos por ação variam 0,021734 , ou seja, há uma expectativa de que um aumento daquela provoque um aumento no valor desses.

Em análise dos dados da Tabela 5, verifica-se que também foi feita a estimação de regressão por pesos nas cross-sections, pelo método dos Mínimos Quadrados Generalizados, mas, considerando a equação $\mathrm{PO}=\mathrm{B}_{0}+\mathrm{B}_{1} \mathrm{CA}+\varepsilon$, ou seja, CA como variável independente, e PO como variável dependente.

Ressalta-se que o poder explicativo da regressão resultante $\left(R^{2}\right)$ é de $48,44 \%$, considerando-se um nível de significância de 5\%, ou seja, a concentração acionária explica $48,44 \%$ do comportamento do payout (Dividendos propostos na DOAR $\div$ Lucro Líquido do

\footnotetext{
${ }^{2} \mathrm{Na}$ análise de dados em painel, foram excluídas as empresas que não possuíam dados (missing values), para todas as variáveis.
} 
Exercício). Além disso, verifica-se que a regressão e os seus coeficientes são, estatisticamente, significantes ( $\alpha$ igual a $5 \%$ ).

Tabela 5: Output para regressão com efeito combinado pelo método GLS, considerando Payout (PO) como a variável dependente

\begin{tabular}{|c|c|c|c|c|}
\hline \multicolumn{4}{|c|}{ Dependent Variable: $\mathrm{PO} ?$} & \\
\hline \multicolumn{4}{|c|}{ Method: Pooled EGLS (Cross-section weights) } & \\
\hline \multicolumn{3}{|l|}{ Sample: 19982005} & & \\
\hline \multicolumn{3}{|c|}{ Included observations: 8} & & \\
\hline \multicolumn{3}{|c|}{ Cross-sections included: 409} & & \\
\hline \multicolumn{4}{|c|}{ Total pool (unbalanced) observations: 2578} & \\
\hline \multicolumn{5}{|c|}{ Linear estimation after one-step weighting matrix } \\
\hline \multicolumn{5}{|c|}{ White diagonal standard errors \& covariance (d.f. corrected) } \\
\hline \multicolumn{5}{|c|}{ Cross sections without valid observations dropped } \\
\hline Variable & Coefficient & Std. Error & t-Statistic & Prob. \\
\hline C & 69.41004 & 1.896285 & 36.60317 & 0.0000 \\
\hline \multirow[t]{2}{*}{ CA? } & -0.659473 & 0.020382 & -32.35591 & 0.0000 \\
\hline & \multicolumn{2}{|c|}{ Weighted Statistics } & & \\
\hline R-squared & 0.484448 & \multicolumn{2}{|c|}{ Mean dependent var } & 544.3155 \\
\hline Adjusted R-squared & 0.484248 & \multicolumn{2}{|c|}{ S.D. dependent var } & 908.0847 \\
\hline S.E. of regression & 652.1493 & \multicolumn{2}{|c|}{ Sum squared resid } & $1.10 \mathrm{E}+09$ \\
\hline F-statistic & 2420.583 & \multicolumn{2}{|c|}{ Durbin-Watson stat } & 1.129057 \\
\hline Prob(F-statistic) & 0.000000 & & & \\
\hline & & & & \\
\hline
\end{tabular}

Um fato interessante a ser observado na Tabela 5 é: se a concentração acionária varia em uma unidade, o payout varia $-0,659473$, ou seja, há uma expectativa de que um aumento daquela provoque uma diminuição significativa no percentual desse.

\section{CONSIDERAÇÕES FINAIS}

Este trabalho utilizou os pressupostos da Teoria de Agência, para verificar as relações existentes entre principal e agente; e os conflitos que podem surgir dessa relação. Sob a luz dessa teoria, buscou-se a evidência de sua aplicabilidade à política de dividendos das empresas brasileiras com ações negociadas na Bovespa.

De acordo com a Teoria de Agência, é muito difícil garantir que o agente tome as melhores decisões do ponto de vista do principal, pois esse agente sempre procura maximizar sua utilidade. No entanto, se o administrador de uma empresa for o seu único proprietário, supõe-se que ele procure maximizar a riqueza da empresa (ou a sua própria), 
ou seja, o administrador-proprietário cumpre a principal meta do administrador financeiro de maximizar a riqueza dos acionistas (no caso, ele mesmo, aumentando o patrimônio da empresa e, conseqüentemente, valorizando o valor de suas ações).

Com base nesses pressupostos, considerou-se, neste trabalho, a hipótese de que "no Brasil, a concentração do controle acionário das empresas influencia a distribuição de dividendos".

Os resultados obtidos, por meio de testes estatísticos, sugerem a não rejeição dessa hipótese. Verificou-se que um aumento na concentração acionária provoca um aumento no valor dos dividendos pagos por ação (ON). Entretanto, há uma expectativa de que um aumento daquela provoque uma diminuição significativa no percentual do payout.

Constataram-se indícios de que a composição acionária possa explicar, em partes, a política de dividendos adotada pelas empresas analisadas. Interessante observar que a concentração acionária provoca uma diminuição no payout (Dividendos propostos $\div$ Lucro Líquido do Exercício), mas quando os dividendos são, efetivamente, pagos, o valor deles por ação aumenta.

Esses resultados confirmam que, no Brasil, "há uma tendência das empresas reterem a maior parcela possível dos lucros" (PROCIANOY, 1994) e que as empresa são concentradas nas mãos de poucos (LOSS, 2004). Segundo esses autores, a retenção dos lucros se deve à escassez de recursos de longo prazo.

Outros fatores podem influenciar os resultados empíricos, como por exemplo, a regulamentação da política de dividendos que não foi considerada como variável neste trabalho. Como a legislação brasileira (Lei 6.404/76 e, posteriormente, Lei 10.303/01) estabelece um dividendo mínimo obrigatório a ser distribuído, a administração não tem total liberdade para decidir sobre a política de distribuição que deverá ser adotada. Além disso, a Lei 9.249/95 introduziu os Juros sobre o Capital Próprio (JSCP), limitados à Taxa de Juros a Longo Prazo (TJLP) e considerados como despesas financeiras e dedutíveis, para fins de imposto de renda e contribuição social. Portanto, em futuras pesquisas, sugere-se considerar essas variáveis.

Os conflitos de interesse decorrentes das relações entre agente e principal possibilitam muitas pesquisas empíricas, ainda pouco exploradas no Brasil.

\section{REFERÊNCIAS}

BERLE, Adolf A.; MEANS, Gabardine C. The Modern Corporation and Private Property. New York, Macmillan Publishing Co., 1932.

BRAV, A. et.al. Payout policy in the 21st century. Journal of Financial Economics, v. 77, 2005, p. 483-527.

BRIGHAM, Eugene F.; GAPENSKI, Louis C.; EHRHARDT, Michael C. Administração financeira: teoria e prática. São Paulo: Atlas, 2001.

EISENHARDT, Kathleen M. Agency theory: an assessment and review. Academy of Management Review, v. 14, n. 1, 1989. p.57-74.

GORDON, M. J. Optimal investiment and financing policy. The Journal of finance. v. 18, n.2, p.264-272, mai. 1963.

GUJARATI, Damodar. Econometria básica. Tradução 4. ed. Rio de Janeiro: Campus, 2006. 
HENDRIKSEN, Eldon S.; VAN BREDA, Michael F. Teoria da contabilidade. São Paulo: Atlas, 1999.

JENSEN, Michael C.; MECKLING, William H. Theory of the firm: managerial behavior, agency costs and ownership structure. Journal of Financial Economics, October 1976.

LA PORTA, R., et al.. Agency problems and dividend policies. The Journal of Finance, v. LV, n. 1, p. 01-33, fev. 2000.

LINTNER, J. Dividends, earnings, leverage, stock prices and the supply of capital to corporations. The Review Economics and Statistics. v. 44, n.3, p.243-269, ago. 1962.

LOPES, Alexsandro Broedel. A relevância da informação contábil para o mercado de capitais: o modelo de Ohlson aplicado à BOVESPA. Tese [Doutorado em Controladoria e Contabilidade]. São Paulo - Faculdade de Economia, Administração e Contabilidade da Universidade de São Paulo, 2001.

- A informação contábil e o mercado de capitais. São Paulo: Pioneira, 2002.

LOSS, Lenita. O inter-relacionamento entre políticas de dividendos e de investimentos: estudo aplicado às companhias brasileiras. Vitória, 2004. Dissertação de Mestrado FUCAPE.

LOSS, Lenita; SARLO NETO, Alfredo. Política de dividendos, na prática, é importante? Revista de Contabilidade e Finanças, São Paulo, Edição Comemorativa, p. 39-53, out. 2003.

MARQUES, Luís David. Modelos dinâmicos com dados em painel: revisão de literatura. Portugal: Faculdade de Economia do Porto, 2000.

MODIGLIANI, F; MILLER, M. H. . Dividend policy, growth, and the valuation of shares. The Journal of Business, v. XXXIV, n. 4, p. 411-433, Oct. 1961.

PROCIANOY, J. L. Conflitos de agência entre controladores e minoritários nas empresas brasileiras negociadas na bolsa de valores de São Paulo: evidências através do comportamento da política de dividendos após as modificações tributárias ocorridas entre 1988-1989. 1994. Tese (Doutorado em Ciências Contábeis) - Departamento de Contabilidade e Atuária, Faculdade de Economia, Administração e Contabilidade, Universidade de São Paulo.

ROSS, Stephen A. The economic theory of agency: the principal's problem. The American Economic Review, v. 63, n. 2, may 1973, p. 134-139.

\section{ENDEREÇO DOS AUTORES}

Fundação Instituto Capixaba de Pesquisa em Contabilidade, Economia e Finanças Av. Fernando Ferrari, 1358 - Goiabeiras Vitoria, ES - Brasil 29075-010
Universidade de São Paulo

Faculdade de Economia Administração e Contabilidade Departamento de Contabilidade Atuária Av. Prof. Luciano Gualberto, 908 - Butantã Sao Paulo, SP - Brasil 05508-900 